\title{
Editorial
}

\section{The Acute Management of Pediatric Heart Failure}

In this thematic issue the spectrum of pediatric heart failure is discussed, providing a "state of the art review" of this complex and challenging disease. Congenital and acquired heart disease, acute and chronic heart failure, organ system interactions and the latest in treatment strategies are reviewed, providing a comprehensive review of a disease that impacts the entire spectrum of health care providers.

The pathophysiology of heart failure is discussed with an emphasis on those factors and challenges that are unique to children, including heart failure in patients with underlying structural heart disease. Cardiomyopathies are thoroughly discussed, including aspects of their presentation and management that are specific to children. Perioperative management strategies for patients following surgery for congenital heart disease are reviewed, including the adverse impact of cardiopulmonary bypass on neonates and children; those factors responsible for neonates and infants having diminished cardio-

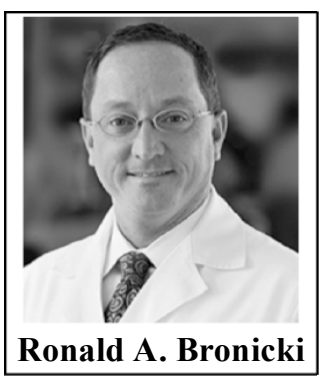
pulmonary reserve compared with older children; unique and challenging aspects of correcting congenital cardiac defects and their impact on post-operative management; and management strategies for patients with single ventricle physiology.

The importance of interactions between the respiratory and cardiovascular systems cannot be overstated, and the intricate role these interaction play in the pathophysiology and treatment of acute and chronic heart failure is discussed. The crosstalk between the kidneys and cardiovascular system represent another important interaction between organ systems and is extensively reviewed, including perioperative factors that may contribute to the development and or progression of renal disease and the cardiorenal syndromes. In addition, the diagnosis, stratification and treatment of acute kidney injury will be discussed. Current hemodynamic monitoring modalities, including the use of near infrared spectroscopy and transpulmonary thermodilution are covered. Pharmacologic agents to treat low cardiac output and vascular failure are discussed, including treatment strategies to optimize cardiac output and systemic oxygen delivery in the unique circulation of patients with single ventricle physiology.

An area of particular excitement and anticipation in pediatric heart failure is the evolution of ventricular assist devices in children. In addition to the successful application of devices designed for adults to larger children, the advent of ventricular assist devices specifically designed for children has provided the pediatric community an option for our smallest patients. Currently available ventricular assists devices that may be deployed in children are discussed, including evolving strategies that impact the timing of their deployment and management.

This review series aims to improve the understanding of the pathophysiology and treatment of cardiovascular disease in children for a wide spectrum of clinicians. A historical perspective and the available evidence to support current practice strategies will be provided as well as aspects of pediatric heart failure that are currently being evaluated.

I would like to acknowledge my administrative assistant Lindsey Gurganious for her immeasurable support in my role as a guest editor and in the collation and submission of the manuscripts to the publisher.

Invited Reviewers: Dr. Catherine Krawczeski, Dr. Daniel J. Penny, Dr. Darren Klugman, Dr. David Axelrod, Dr. Douglas Moodie, Dr. Javier Lassa, Dr. Jeff Heinle, Dr. Mary Taylor, Dr. Mary McBride, Dr. Michele Domico.

Dr. Ronald A. Bronicki

(Guest Editor)

Baylor College of Medicine

Texas Children's Hospital

Houston, Texas 77037

USA

Tel: 832.826.6214

Fax: 832.825.7422

E-mail: Bronicki@bcm.edu 